\title{
Quantum control for neutrons in nuclear reaction
}

\author{
Quan-Fang Wang \\ ${ }^{1}$ Mechanical and Automation Engineering, The Chinese University of Hong Kong, \\ Shatin, N.T.,Hong Kong \\ E-mail: quanfangwang@hotmail.com
}

\begin{abstract}
Quantum control of neutrons in nuclear reaction in considered in this work. Neutrons fission from uranium ${ }^{235} \mathrm{U}$ of chain reaction is interested to be controlled as target theoretically. Control theory is applied to interacted many-body neutrons collision in the framework of variational method. Full proof is provided for quantum optimal control of scattered poly-neutrons.
\end{abstract}

PACS numbers: 02.30.Yy, 24.00.Cn, 25.85.Ec, 33.20.Tp

Keywords: Quantum control, neutrons, Klein-Gordon-Schrödinger equation, scatter, chain reaction

Submitted to: J. Phys. A: Math. Gen. 


\section{Introduction}

Quantum control at nuclei scale is interesting topic in the field of particle physics and quantum mechanics. Specially, the status of neutrons in nuclear reaction is a sensitivity as particle lasting of half century. People pay the fatal price for the gain and pain of neutrons reaction. Except the surprising of the chargeless (natural), neutron, particle in a nucleus, the impact of neutrons fission is breaking the force of holding elementary particles together in atoms, releasing huge energy and producing large number of neutrons to make chain reaction incident. Obviously, to do control as nuclear reaction is extremely important thing from past years to now. In fact, neutrons chain reaction is a wonderful chance to make such a control dream come true. Physically, it had being worked at existing nuclear reactors for various aims (power plants, rocket launch, manmade satellite). Control take place of neutrons in nuclear reaction is not just for nuclear bomb and nuclear reactors, it had been extending to utilize the nuclear energy and nuclear power in present society and academic level purpose.

Control methods at nuclear-scale scatter in a variety of aspects: for instance, temperature adjustment; increasing or decreasing pressure; appendix chemical reagent (insert proper rods at reaction fuel); quantitative confinements (control reacted matter); reducing energy emission (fit the practical needs); and so forth. Not limited to qualitative investigation for neutrons reaction and nuclear chain reaction. Much more quantitative works need to do theoretically, computationally, and experimentally. At the point of view in control field, nuclear reaction is considered as quantum system control using quantum mechanics, and control theory. It is an attempt to work out for doing nuclear control systematically. At current experimental equipments, it is difficult to track one particle as it react or collide to other particle. Not lost of generality, the real goal is to manipulate the large volume of particles for occurred chain reaction (no harmness, no explosion).

It is interesting for us to pick up neutrons in nuclear reaction as the control objective to apply quantum control theory. The task is try to establish a physical control model of neutrons chain reactions. Find a reasonable control theory for neutrons in the meaning of quantum physics and control areas.

This paper is divided into a couple of sections. In section 2, control model for neutrons fission is proposed in the view point of physics. Section 3 is to supply full control theory for many-body neutrons in nuclear reaction. Section 4 is Bang-Bang principle. Section 5 is concluding remark.

\section{Control model for neutrons fission}

Neutrons in nuclear chain reaction of uranium ${ }^{235} \mathrm{U}$ is taking account into control (cf. [15]). For many-body neutrons to establish a rational physical model of quantum controlling. Let $n$ be the numbers of involved neutrons at a chain reaction initially, and $i, j$ denote positive integers $1 \leq i \leq n, 1 \leq j \leq n$. Suppose control at $n$ neutrons 
are different, and recognized as $n$ controls. It means that each particle receive different force from entire control source (inserted rod, temperature, pressure, reagent). As usual, denote $M$ is mass of neutron, $\hbar$ is reduced Planck constant and $\mathbf{i}$ denote the imaginary part of complex function. Let $\Omega$ be an open bounded set of $\mathbf{R}^{3}$ and $Q=(0, T) \times \Omega$ for $T>0$. Thereby, for $(t, \mathbf{x}) \in Q, n$-body neutrons is satisfying Schrödinger (KGS) equations (cf. [6]) simultaneously as

$$
\left\{\begin{array}{c}
\mathbf{i} \hbar \frac{\partial \psi^{1}}{\partial t}+\frac{\hbar^{2}}{2 M} \frac{\partial^{2} \psi^{1}}{\partial \mathbf{x}^{2}}+\mathbf{i} \alpha \psi^{1}+\mu \sum_{i=1}^{n} \psi^{i} \psi^{1}+u^{1} \psi^{1}=0 \\
\vdots \\
\mathbf{i} \hbar \frac{\partial \psi^{n}}{\partial t}+\frac{\hbar^{2}}{2 M} \frac{\partial^{2} \psi^{n}}{\partial \mathbf{x}^{2}}+\mathbf{i} \alpha \psi^{n}+\mu \sum_{i=n}^{n} \psi^{i} \psi^{n}+u^{n} \psi^{n}=0 \\
\left.\left(\psi^{1}, \cdots, \psi^{n}\right)\right|_{t=0}=\left(\psi_{0}^{j}, \cdots, \psi_{0}^{n}\right) .
\end{array}\right.
$$

In here, $\psi^{j}(t, \mathbf{x})$, complex-valued functions, represent probability amplitudes of neutron fields. $u^{j}, j=1,2, \ldots, n$ are control inputs at neutrons, it is corresponding to external force in nuclear reaction. Such as, control rod for nuclear fuel in a nuclear reactor. Assume the $i$-th neutron $\psi^{i}$ interact to $j$-th neutron $\psi_{j}$, and coupling constant is $\mu$, and $i \neq j . \quad \alpha$ is coefficient of dissipative or scattered term. Denote $\boldsymbol{\psi}=\left(\psi^{1}, \psi^{2}, \cdots, \psi^{n}\right)$, $\boldsymbol{u}=\left(u^{1}, u^{2}, \cdots, u^{n}\right)$ and analogous notations, then multi-Schrödinger system (1) is in the form of

$$
\left\{\begin{array}{l}
\mathbf{i} \hbar \frac{\partial \boldsymbol{\psi}}{\partial t}+\frac{\hbar^{2}}{2 M} \frac{\partial^{2} \boldsymbol{\psi}}{\partial \mathbf{x}^{2}}+\mathbf{i} \alpha \boldsymbol{\psi}+\mu \boldsymbol{\alpha}(\boldsymbol{\psi})+\boldsymbol{\sigma}(\boldsymbol{u}, \boldsymbol{\psi})=0 \\
\boldsymbol{\psi}(0)=\boldsymbol{\psi}_{0}
\end{array}\right.
$$

In (2), coupling term $\boldsymbol{\alpha}(\boldsymbol{\psi})=\left(\sum_{i=1}^{n} \psi^{i} \psi^{1}, \cdots, \sum_{i=1}^{n} \psi^{i} \psi^{n}\right)^{T}$, external force $\boldsymbol{\sigma}(\boldsymbol{u}, \boldsymbol{\psi})=$ $\left(u^{1} \psi^{1}, \cdots, u^{n} \psi^{n}\right)^{T}, \quad \boldsymbol{\psi}(0)=\left(\psi^{1}(0), \cdots, \psi^{n}(0)\right)$ and $\boldsymbol{\psi}_{0}=\left(\psi_{0}^{1}, \cdots, \psi_{0}^{n}\right)$. MultiSchrödinger system (1) represents many-body interactions of neutrons [15]. The equation in system (2) is said as neutrons equation.

\section{Control theory for neutrons chain reaction}

At first, citing $[4,7]$ to do mathematical setting. For complex-valued function $\psi^{j}$, define complex spaces $\mathbb{L}^{2}(\Omega)$ and $\mathbb{H}_{0}^{1}(\Omega)$ corresponding to real spaces $L^{2}(\Omega)$ and $H_{0}^{1}(\Omega)$, respectively (cf. [1, 14]). Let $\mathbb{L}^{2}(\Omega)=\left\{\psi^{j} \mid \psi^{j}=\psi^{1 j}+\mathbf{i} \psi^{2 j}, \psi^{1 j}, \psi^{2 j} \in L^{2}(\Omega)\right\}$, and $\mathbb{H}_{0}^{1}(\Omega)=\left\{\psi^{j} \mid \psi^{j}=\psi^{1 j}+\mathbf{i} \psi^{2 j}, \psi^{1 j}, \psi^{2 j} \in H_{0}^{1}(\Omega)\right\}$. Define norm of $\psi^{j}$ in $\mathbb{L}^{2}(\Omega)$ as $\left\|\psi^{j}\right\|_{\mathbb{L}^{2}(\Omega)}=\left(\left\|\psi^{1 j}\right\|_{L^{2}(\Omega)}^{2}+\left\|\psi^{2 j}\right\|_{L^{2}(\Omega)}^{2}\right)^{\frac{1}{2}}$. If $\psi^{j^{\prime}}=\psi^{1 j^{\prime}}+\mathbf{i} \psi^{2 j^{\prime}} \in \mathbb{L}^{2}(\Omega)$, define the inner product to $\psi^{j}$ in complex space $\mathbb{L}^{2}(\Omega)$ by

$$
\left(\psi^{j}, \psi^{j^{\prime}}\right)_{\mathbb{L}^{2}(\Omega)}=\left(\left(\psi^{1 j}, \psi^{1 j^{\prime}}\right)_{L^{2}(\Omega)}+\left(\psi^{2 j}, \psi^{2 j^{\prime}}\right)_{L^{2}(\Omega)}\right)+\mathbf{i}\left(\left(\psi^{2 j}, \psi^{1 j^{\prime}}\right)_{L^{2}(\Omega)}-\left(\psi^{1 j}, \psi^{2 j^{\prime}}\right)_{L^{2}(\Omega)}\right) .
$$


Define norm of $\mathbb{H}_{0}^{1}(\Omega)$ as $\left\|\psi^{j}\right\|_{\mathbb{H}_{0}^{1}(\Omega)}=\left(\left\|\psi^{1 j}\right\|_{H_{0}^{1}(\Omega)}^{2}+\left\|\psi^{2 j}\right\|_{H_{0}^{1}(\Omega)}^{2}\right)^{\frac{1}{2}}$. If $\psi^{j^{\prime}} \in \mathbb{H}_{0}^{1}(\Omega)$, define the inner product to $\psi^{j}$ in complex space $\mathbb{H}_{0}^{1}(\Omega)$ as

$$
\left(\psi^{j}, \psi^{j^{\prime}}\right)_{\mathbb{H}_{0}^{1}(\Omega)}=\left(\psi^{1 j}, \psi^{1 j^{\prime}}\right)_{H_{0}^{1}(\Omega)}+\left(\psi^{2 j}, \psi^{2 j^{\prime}}\right)_{H_{0}^{1}(\Omega)} .
$$

Set $\mathbb{H}=\mathbb{L}^{2}(\Omega), \mathbb{V}=\mathbb{H}_{0}^{1}(\Omega), \mathbb{V}^{\prime}$ is complex conjugate space of $\mathbb{V}$. In fact, $(\mathbb{V}, \mathbb{H})$ is a complex Gelfand triple spaces $\mathbb{V} \hookrightarrow \mathbb{H} \hookrightarrow \mathbb{V}^{\prime}$, two embeddings are continuous, dense and compact.

Definition 1 Define weak solution space by Hilbert space:

$$
W(0, T)=\left\{\boldsymbol{\psi} \mid \boldsymbol{\psi} \in L^{2}(0, T ; \mathbb{V})^{n}, \boldsymbol{\psi}^{\prime} \in L^{2}\left(0, T ; \mathbb{V}^{\prime}\right)^{n}\right\}
$$

The norm of complex solution space $W(0, T)$ is defined by

$$
\|\boldsymbol{\psi}\|_{W(0, T)}=\left(\|\boldsymbol{\psi}\|_{L^{2}(0, T ; \mathbb{V})^{n}}^{2}+\left\|\boldsymbol{\psi}^{\prime}\right\|_{L^{2}\left(0, T ; \mathbb{V}^{\prime}\right)^{n}}^{2}\right)^{\frac{1}{2}} .
$$

For $\boldsymbol{\psi}=\left(\psi^{1}, \psi^{2}, \cdots, \psi^{n}\right), \boldsymbol{\varphi}=\left(\varphi^{1}, \varphi^{2}, \cdots, \varphi^{n}\right) \in W(0, T)$, their inner product is

$$
(\boldsymbol{\psi}, \boldsymbol{\varphi})_{W(0, T)}=(\boldsymbol{\psi}, \boldsymbol{\varphi})_{L^{2}(0, T ; \mathbb{V})^{n}}+\left(\boldsymbol{\psi}^{\prime}, \boldsymbol{\varphi}^{\prime}\right)_{L^{2}\left(0, T ; \mathbb{V}^{\prime}\right)^{n}}
$$

Definition 2 Neutrons $\boldsymbol{\psi}$ is said weak solution of many-body neutrons system (2) if $\boldsymbol{\psi} \in W(0, T)$ and satisfy weak form

$\left\{\begin{array}{l}\int_{0}^{T} \int_{\Omega}\left[-\mathbf{i} \hbar \boldsymbol{\psi} \frac{\partial \overline{\mathrm{n}}}{\partial t}-\frac{\hbar^{2}}{2 M} \frac{\partial \boldsymbol{\psi}}{\partial \mathbf{x}} \frac{\partial \overline{\mathrm{n}}}{\partial \mathbf{x}}+\mathbf{i} \alpha \boldsymbol{\psi} \overline{\mathrm{n}}+\mu \boldsymbol{\alpha}(\boldsymbol{\psi}) \overline{\mathrm{n}}+\boldsymbol{\sigma}(\boldsymbol{u}, \boldsymbol{\psi}) \overline{\mathrm{n}}\right] d \mathbf{x} d t=\int_{\Omega} \mathbf{i} \hbar \boldsymbol{\psi}_{0} \overline{\mathrm{n}}(0) d \mathbf{x} \\ \boldsymbol{\psi}(0)=\boldsymbol{\psi}_{0}\end{array}\right.$

for all $\overline{\mathrm{n}}$ is conjugate of $\mathrm{n} \in C^{1}(0, T ; \mathbb{V})$ and $\mathrm{n}(T)=0$.

Denote quantum optimal control by $\boldsymbol{u}^{*}(t, \mathbf{x})=\left(u^{1 *}(t, \mathbf{x}), u^{2 *}(t, \mathbf{x}), \cdots, u^{n *}(t, \mathbf{x})\right)$. Suppose $\mathcal{U}$ is space of external control $\boldsymbol{u}$, control is depended on spatial variable $\mathbf{x}$ and time $t$. Let $\mathcal{U}_{a d}$ be a non-empty closed convex (bounded) admissible subset of $\mathcal{U}$. For the time-varying particle, it is possible to track the terminal value as final observation. Therefore, the criteria function for many-body neutrons system (2) can be taken the form of

$$
J(\boldsymbol{u})=\epsilon^{1}\left\|\boldsymbol{\psi}_{f}(\boldsymbol{u})-\boldsymbol{\psi}_{\text {target }}\right\|_{\mathbb{V}}^{2}+\epsilon^{2}(\boldsymbol{u}, \boldsymbol{u})_{\mathcal{U}}, \quad \forall \boldsymbol{u} \in \mathcal{U}_{a d}
$$

and $(\boldsymbol{u}, \boldsymbol{u})_{\mathcal{U}}=\sum_{j=1}^{n}\left(u^{j}, u^{j}\right) \mathcal{U}$. In cost function (4), $\boldsymbol{\psi}_{\text {target }}=\boldsymbol{\psi}_{\text {target }}^{1}+\mathbf{i} \boldsymbol{\psi}_{\text {target }}^{2} \in \mathbb{V}$ is target state, and $\boldsymbol{\psi}_{f}(\boldsymbol{u})=\boldsymbol{\psi}_{f}^{1}(\boldsymbol{u})+\mathbf{i} \psi_{f}^{2}(\boldsymbol{u}) \in \mathbb{V}$ is observed final state at time $t_{f}$. Then

$$
J(\boldsymbol{u})=\epsilon^{1}\left(\left\|\boldsymbol{\psi}_{f}^{1}(\boldsymbol{u})-\boldsymbol{\psi}_{\text {target }}^{1}\right\|_{H_{0}^{1}(\Omega)}^{2}+\left\|\boldsymbol{\psi}_{f}^{2}(\boldsymbol{u})-\boldsymbol{\psi}_{\text {target }}^{2}\right\|_{H_{0}^{1}(\Omega)}^{2}\right)+\epsilon^{2}(\boldsymbol{u}, \boldsymbol{u})_{\mathcal{U}}
$$

In here, $\epsilon^{1}, \epsilon^{2}$ are weighted coefficients for balancing the values of two inherent costs and one running cost.

Cite [4] and weak form (3) to prove the existence Theorem. For $j=1$ and neutron in Yukawa interaction, refer [8] for one nucleon and one meson case. In here is to develop the variational theory to many-body neutrons in complex Hilbert space. 
Theorem 1 For $\boldsymbol{\psi}_{0} \in \mathbb{V}^{n}$, then there exists a unique weak solutions $\boldsymbol{\psi}$ of many-body neutrons quantum system (1).

Proof. Cite [3], using Faedo-Galerkin method (cf. [5]) for existence of weak solution. Construct an approximate solution for many-body neutrons system (1). Since $\mathbb{V} \hookrightarrow \mathbb{H}$ is compact, then there exists orthogonal basis of $\mathbb{H},\left\{w^{i}\right\}_{i=1}^{\infty}$ consisting of eigenfunctions of $A=\Delta$, such that $A w^{i}=\lambda^{i} w^{i}$ for all $i, 0<\lambda^{1} \leq \lambda^{2} \leq \cdots, \lambda^{j} \rightarrow \infty$ as $i \rightarrow \infty$. Denote by $P_{n^{\prime}}$ the orthogonal projection of $\mathbb{H}$ (or $\mathbb{V}$ ) onto the space spanned by $\left\{w^{1}, \cdots, w^{n^{\prime}}\right\}$. For each $n^{\prime} \in N$, an approximate solution for multi-Schrödinger system (1) can be expressed by $\psi_{n^{\prime}}^{j}(t)=\sum_{i=1}^{n^{\prime}} a_{i j}(t) w^{i}$, where $a_{i j}(t)$ is complex-valued coefficient function. Denote $\mathbf{w}=\left(w^{1}, w^{2}, \cdots, w^{n^{\prime}}\right)^{T}$, then approximate complex-valued solution $\boldsymbol{\psi}_{n^{\prime}}(t)$ satisfy a ordinary differential equation given by

$$
\left\{\begin{array}{l}
\int_{\Omega}\left[\mathbf{i} \hbar \frac{\partial \boldsymbol{\psi}_{n^{\prime}}}{\partial t} \overline{\mathbf{w}}-\frac{\hbar^{2}}{2 M} \frac{\partial \boldsymbol{\psi}_{n^{\prime}}}{\partial \mathbf{x}} \frac{\partial \overline{\mathbf{w}}}{\partial \mathbf{x}}+\mathbf{i} \alpha \boldsymbol{\psi}_{n^{\prime}} \overline{\mathbf{w}}+\mu \boldsymbol{\alpha}\left(\boldsymbol{\psi}_{n^{\prime}}\right) \overline{\mathbf{w}}\right] d \mathbf{x} d t=\int_{\Omega} \boldsymbol{\sigma}\left(\boldsymbol{u}, \boldsymbol{\psi}_{n^{\prime}}\right) \mathbf{w} d \mathbf{x} \\
\boldsymbol{\psi}_{n^{\prime}}(0)=\boldsymbol{\psi}_{0} .
\end{array}\right.
$$

In here, $\overline{\mathbf{w}}$ is conjugate of $\mathbf{w}$ in dual space $\mathbb{H}^{n}$. Standard theory of ODE ensure that many-body neutrons system exist an unique local solution $\boldsymbol{\psi}_{n^{\prime}}$ for $n^{\prime} \in N$. For $\boldsymbol{\psi}_{0} \in \mathbb{V}^{n}$, there exists $\boldsymbol{\psi}_{0}^{n^{\prime}} \in \mathbb{H}_{0}^{1}(\Omega)^{n}$ such that

$$
\boldsymbol{\psi}_{0}^{n^{\prime}} \rightarrow \boldsymbol{\psi}_{0} \text { strongly in } \mathbb{H}_{0}^{1}(\Omega)^{n} \text {. }
$$

For $\boldsymbol{\psi}_{0}^{n^{\prime}}$, approximate solution $\boldsymbol{\psi}_{n^{\prime}}$ is existing, $1 \leq n^{\prime} \leq N$. The argument implies that $\boldsymbol{\psi}_{n^{\prime}}$ is bounded in $L^{\infty}(0, T ; \mathbb{V})^{n}$. For $j$-th neutron $\psi^{j}$, assume $\psi^{j}$ and $\psi^{j^{\prime}}$ are two solutions of $(1)$ to initials $\psi_{0}^{j}$ and $\psi_{0}^{j^{\prime}}$. Calculate estimate

$$
\frac{d}{d t} G^{j j^{\prime}}(t)+\mathbf{i} \alpha\left\|\psi^{j}-\psi^{j^{\prime}}\right\|_{\mathbb{H}}^{2} \leq \mu\left(\sum_{i=1}^{n}\left\|\psi^{i}\right\|_{\mathbb{H}}^{2}+1 / p\right)\left\|\psi^{j}-\psi^{j^{\prime}}\right\|_{\mathbb{H}}^{2}+1 / p^{\prime}\left\|u^{j}\right\|_{\mathbb{H}}^{2}, 1 / p+1 / p^{\prime}=1
$$

where $G^{j j^{\prime}}(t)=\frac{1}{2}\left\|\psi_{t}^{j}(t)-\psi_{t}^{j^{\prime}}(t)\right\|_{\mathbb{H}}^{2}+\frac{\hbar^{2}}{2 M}\left\|\psi^{j}(t)-\psi^{j^{\prime}}(t)\right\|_{\mathbb{H}}^{2}$. It means that

$$
\frac{d}{d t} G^{j j^{\prime}}(t) \leq C(t) G^{j j^{\prime}}(t)+F(t)
$$

for $C(t)=\max \left\{\frac{1}{2}, \mu \sum_{i=1}^{n}\left\|\psi^{i}\right\|_{\mathbb{H}}^{2}+1 / p\right\}, F(t)=1 / p^{\prime}\left\|u^{j}\right\|_{\mathbb{H}}^{2}$. From Gronwall inequality to yield $G^{j j^{\prime}}(t) \rightarrow 0$ as $j, j^{\prime} \rightarrow \infty$. That is $\boldsymbol{\psi}_{n^{\prime}} \rightarrow \boldsymbol{\psi}$ in $C\left(0, T ; \mathbb{L}^{2}(\Omega)\right)^{n}$ as $n^{\prime} \rightarrow \infty$. It proved Theorem 1 .

Theorem 2 For $\boldsymbol{\psi}_{0} \in \mathbb{V}^{n}$, if $\mathcal{U}_{a d}$ is closed convex (bounded) subset of $\mathcal{U}$. Then there exists at least one quantum optimal control $\boldsymbol{u}^{*}=\left(u^{1 *}, u^{2 *}, \ldots, u^{n *}\right)$ for many-body neutrons system (2) subject to criteria function (4).

Proof. Set $J=\inf _{\boldsymbol{u} \in \mathcal{U}_{a d}} J(\boldsymbol{u}) . \mathcal{U}_{a d}$ is non-empty, there is a sequence $\left\{\boldsymbol{u}_{k}\right\}$ in $\mathcal{U}_{a d}$ such that $\inf _{\mathbf{u} \in \mathcal{U}_{a d}} J(\boldsymbol{u})=\lim _{k \rightarrow \infty} J\left(\boldsymbol{u}_{k}\right)=J$. Clearly, $\left\{J\left(\boldsymbol{u}_{k}\right)\right\}$ is bounded in $\mathbf{R}^{+}$. Since $\mathcal{U}_{a d}$ is 
closed convex (bounded) subset of $\mathcal{U}$, a subsequences $\left\{\boldsymbol{u}_{k^{\prime}}\right\}$ can be extracted from $\left\{\boldsymbol{u}_{k}\right\}$, and $\boldsymbol{u}^{*} \in \mathcal{U}_{a d}$ such that

$$
\boldsymbol{u}_{k^{\prime}} \rightarrow \boldsymbol{u}^{*} \quad \text { weakly in } \mathcal{U} \text { as } k^{\prime} \rightarrow \infty \text {. }
$$

For $\boldsymbol{\psi}$ and $\boldsymbol{u}$, the boundedness of $\mathcal{U}_{a d}$ and [7] to get that $\boldsymbol{\psi}(\boldsymbol{u})$ is bounded at $W(0, T)$. Setting $\boldsymbol{\psi}^{*}=\boldsymbol{\psi}\left(\boldsymbol{u}^{*}\right)$, there exist a subsequence $\boldsymbol{\psi}\left(\boldsymbol{u}_{k^{\prime}}\right)$ of $\boldsymbol{\psi}\left(\boldsymbol{u}_{k}\right)$, and $\boldsymbol{\psi}^{*} \in W(0, T)$ such that

$$
\boldsymbol{\psi}\left(\boldsymbol{u}_{k^{\prime}}\right) \rightarrow \boldsymbol{\psi}^{*} \quad \text { weakly in } W(0, T)
$$

as $k^{\prime} \rightarrow \infty$. Since the embedding $\mathbb{V} \hookrightarrow \mathbb{H}$ is compact, from Aubin-Lions-Temam compactness embedding theorem (cf. [7]), there is $\overline{\boldsymbol{\psi}}$ that

$$
\boldsymbol{\psi}\left(\boldsymbol{u}_{k^{\prime}}\right) \rightarrow \overline{\boldsymbol{\psi}} \text { strongly in } L^{2}(0, T ; \mathbb{H})^{n}
$$

as $k^{\prime} \rightarrow \infty$. Then to get convergences for $\boldsymbol{\psi}$ as $k^{\prime} \rightarrow \infty$ :

$$
\begin{array}{ll}
\boldsymbol{\psi}_{t}\left(\boldsymbol{u}_{k^{\prime}}\right) \rightarrow \overline{\boldsymbol{\psi}}_{t} & \text { weakly in } L^{2}\left(0, T ; \mathbb{V}^{\prime}\right)^{n}, \\
\nabla \boldsymbol{\psi}\left(\boldsymbol{u}_{k}\right) \rightarrow \nabla \overline{\boldsymbol{\psi}} & \text { weakly in } L^{2}(0, T ; \mathbb{H})^{n},
\end{array}
$$

Set $\psi^{j k^{\prime}}=\psi^{j}\left(\boldsymbol{u}_{k^{\prime}}\right)$ for $j$-th neutron, therefore, from weak from to find

$$
\begin{aligned}
& \int_{0}^{T} \int_{\Omega}\left[-\mathbf{i} \hbar \psi^{j k^{\prime}} \frac{\partial \bar{n}^{j}}{\partial t}-\frac{\hbar^{2}}{2 M} \frac{\partial \psi^{j k^{\prime}}}{\partial \mathbf{x}} \frac{\partial \bar{n}^{j}}{\partial \mathbf{x}}+\mathbf{i} \alpha \psi^{j k^{\prime}} \bar{n}^{j}\right. \\
& \left.+\sum_{i=1}^{i{ }^{\prime}} \psi^{i k^{\prime}} \psi^{j k^{\prime}} \bar{n}^{j}+u^{j k^{\prime}} \psi^{j k^{\prime}} \bar{n}^{j}\right] d \mathbf{x} d t=\int_{\Omega} \mathbf{i} \hbar \psi_{0}^{j} \bar{n}^{j}(0) d \mathbf{x},
\end{aligned}
$$

for all $\bar{n}^{j}$ is conjugate of $n^{j}$ in $C^{1}(0, T ; \mathbb{V})$. If taking $k^{\prime} \rightarrow \infty$ in above formula to yield that

$$
\begin{aligned}
& \int_{0}^{T} \int_{\Omega}\left[-\mathbf{i} \hbar \bar{\psi}^{j} \frac{\partial \bar{n}^{j}}{\partial t}-\frac{\hbar^{2}}{2 M} \frac{\partial \bar{\psi}^{j}}{\partial \mathbf{x}} \frac{\partial \bar{n}^{j}}{\partial \mathbf{x}}+\mathbf{i} \alpha \bar{\psi}^{j} \bar{n}^{j}\right. \\
& \left.+\sum_{i=1}^{n} \bar{\psi}^{i} \bar{\psi}^{j} \bar{n}^{j}+u^{j *} \bar{\psi}^{j} \bar{n}^{j}\right] d \mathbf{x} d t=\int_{\Omega} \mathbf{i} \hbar \psi_{0}^{j} \bar{n}^{j}(0) d \mathbf{x},
\end{aligned}
$$

That is, the limit $\bar{\psi}^{j}$ satisfy weak form for all $\psi^{j} \in \mathbb{V}$ in the sense of distribution $\mathcal{D}^{\prime}(0, T)$ on $(0, T)$. From uniqueness of weak solution for many-body neutrons system (1) that $\bar{\psi}^{j}=\psi^{j}\left(\boldsymbol{u}^{*}\right)$. Namely

$$
\begin{aligned}
& \psi^{j}\left(\boldsymbol{u}_{k^{\prime}}\right) \rightarrow \psi^{j}\left(\boldsymbol{u}^{*}\right) \quad \text { strongly in } \mathbb{L}^{2}(Q), \\
& \psi_{f}^{j}\left(\boldsymbol{u}_{k^{\prime}}\right) \rightarrow \psi_{f}^{j}\left(\boldsymbol{u}^{*}\right) \quad \text { strongly in } \mathbb{L}^{2}(\Omega)
\end{aligned}
$$

as $k^{\prime} \rightarrow \infty$. Since norm $\|\cdot\|_{\mathbb{L}^{2}(\Omega)}$ is lower semi-continuous in weak topology of $\mathbb{L}^{2}(\Omega)$.

$$
\liminf _{k^{\prime} \rightarrow \infty}\left\|\boldsymbol{\psi}_{f}\left(\boldsymbol{u}_{k^{\prime}}\right)-\boldsymbol{\psi}_{\text {target }}\right\|_{\mathbb{V}^{n}}^{2} \geq\left\|\boldsymbol{\psi}_{f}\left(\boldsymbol{u}^{*}\right)-\boldsymbol{\psi}_{\text {target }}\right\|_{\mathbb{V}^{n}}^{2} .
$$

Vice versa, from weak convergence to get

$$
\liminf _{k^{\prime} \rightarrow \infty}\left(\boldsymbol{u}_{k^{\prime}}, \boldsymbol{u}_{k^{\prime}}\right) \mathcal{U} \geq\left(\boldsymbol{u}^{*}, \boldsymbol{u}^{*}\right) \mathcal{U} .
$$

Since $J$ is weakly lower semi-continous, $J=\liminf _{k^{\prime} \rightarrow \infty} J\left(\boldsymbol{u}_{k^{\prime}}\right) \geq J\left(\boldsymbol{u}^{*}\right)$, Thus, $J\left(\boldsymbol{u}^{*}\right)=$ $\inf _{\boldsymbol{u} \in \mathcal{U}_{a d}} J(\boldsymbol{u})$. It means that $\boldsymbol{u}^{*}$ is quantum optimal control of many-body neutrons system (1) subject to criteria function (4). It is finished of Theorem 2. 
Theorem 3 For $\boldsymbol{\psi}_{0} \in \mathbb{V}^{n}$, and assume $\mathcal{U}_{\text {ad }}$ is the closed convex (bounded) admissible subset of $\mathcal{U}$. Quantum optimal control $\boldsymbol{u}^{*}$ for many-body neutrons system (1) subject to cost function (4) is characterized by the Eular-Lagrange system as:

$$
\begin{aligned}
& \left\{\begin{array}{l}
\mathbf{i} \hbar \frac{\partial \boldsymbol{\psi}}{\partial t}+\frac{\hbar^{2}}{2 M} \frac{\partial^{2} \boldsymbol{\psi}}{\partial \mathbf{x}^{2}}+\mathbf{i} \alpha \boldsymbol{\psi}+\mu \boldsymbol{\alpha}(\boldsymbol{\psi})+\boldsymbol{\sigma}\left(\boldsymbol{u}^{*}, \boldsymbol{\psi}\right)=0 \text { in } \mathrm{Q}, \\
\boldsymbol{\psi}(0)=\boldsymbol{\psi}_{0} \text { in } \Omega
\end{array}\right. \\
& \left\{\begin{array}{l}
\mathbf{i} \hbar \frac{\partial \boldsymbol{\nu}}{\partial t}+\frac{\hbar^{2}}{2 M} \frac{\partial^{2} \boldsymbol{\nu}}{\partial \mathbf{x}^{2}}+\mathbf{i} \alpha \boldsymbol{\nu}+\mu \boldsymbol{\alpha}^{*}(\boldsymbol{\psi}) \boldsymbol{\nu}=0 \text { in } \mathrm{Q}, \\
\mathbf{i} \boldsymbol{\nu}(T)=\boldsymbol{\psi}_{f}\left(\boldsymbol{u}^{*}\right)-\boldsymbol{\psi}_{\text {target }} \text { in } \Omega
\end{array}\right. \\
& \left(\boldsymbol{u}^{*}, \boldsymbol{u}-\boldsymbol{u}^{*}\right)_{\mathcal{U}}+\int_{0}^{T} \int_{\Omega} \boldsymbol{\sigma}^{*} \boldsymbol{\nu}\left(\boldsymbol{u}^{*}\right)\left(\boldsymbol{u}-\boldsymbol{u}^{*}\right) d \mathbf{x} d t \geq 0, \quad \boldsymbol{u} \in \mathcal{U}_{a d}
\end{aligned}
$$

In adjoint system (6), $\boldsymbol{\nu} \in W(0, T)$ is solution corresponding to $\boldsymbol{\psi}$ in many-body neutrons system (5), and $\boldsymbol{\alpha}^{*}(\boldsymbol{\psi}), \boldsymbol{\sigma}^{*}$ are conjugate operators of $\boldsymbol{\alpha}, \boldsymbol{\sigma}$. Inequality (7) is necessary optimality condition for $\boldsymbol{u}^{*}$.

\section{Bang-Bang principle}

For system cost, consider Bang-Bang principle from necessary optimality condition (7). For time depended control $\boldsymbol{u} \in \mathcal{U}$, take non-empty bounded control space as admissible subset

$$
\mathcal{U}_{a d}=\left\{\boldsymbol{u} \mid \boldsymbol{u}^{1} \leq \boldsymbol{u}(t, \mathbf{x}) \leq \boldsymbol{u}^{2}, \text { a.e. } t \in[0, T], \mathbf{x} \in \Omega\right\}
$$

In here, $\boldsymbol{u}^{1}, \boldsymbol{u}^{2} \in L^{2}(Q)$, the component of $\boldsymbol{u}, \boldsymbol{u}^{1}, \boldsymbol{u}^{2}$ satisfying $u^{j 1} \leq u^{j} \leq u^{j 2}$ for $1 \leq j \leq n$. For arbitrary solution $\boldsymbol{\nu}=\boldsymbol{\nu}^{1}+\mathbf{i} \boldsymbol{\nu}^{2}$ of adjoint system (6), setting $\boldsymbol{u}=\boldsymbol{u}^{*}$ in (7)

$$
\int_{0}^{T} \int_{\Omega}\left(\boldsymbol{\sigma}^{*}\left(\boldsymbol{\nu}^{1}\left(\boldsymbol{u}^{*}\right)+\boldsymbol{\nu}^{2}\left(\boldsymbol{u}^{*}\right)\right), \boldsymbol{u}-\boldsymbol{u}^{*}\right) d \mathbf{x} d t \geq 0, \quad \forall \boldsymbol{u} \in \mathcal{U}_{a d}
$$

From Lebesgue convergence theorem to attain

$$
\left(\boldsymbol{\sigma}^{*}\left(\boldsymbol{\nu}^{1}\left(\boldsymbol{u}^{*}\right)+\boldsymbol{\nu}^{2}\left(\boldsymbol{u}^{*}\right)\right), \boldsymbol{u}(t)-\boldsymbol{u}^{*}(t)\right)_{L^{2}(Q)} \geq 0 \text { a.e. } t \in[0, T], \mathbf{x} \in \Omega, \forall \boldsymbol{u} \in \mathcal{U}_{a d} .
$$

Then, the property of optimal control $\boldsymbol{u}^{*}$ as

$$
\begin{array}{ll}
\boldsymbol{u}^{*}(t, \mathbf{x})=\boldsymbol{u}^{1} & \text { if } \boldsymbol{\nu}^{1}\left(\boldsymbol{u}^{*}\right)+\boldsymbol{\nu}^{2}\left(\boldsymbol{u}^{*}\right)>0, \text { a.e. } t \in[0, T], \mathbf{x} \in \Omega, \\
\boldsymbol{u}^{*}(t, \mathbf{x})=\boldsymbol{u}^{2} & \text { if } \boldsymbol{\nu}^{1}\left(\boldsymbol{u}^{*}\right)+\boldsymbol{\nu}^{2}\left(\boldsymbol{u}^{*}\right)<0, \text { a.e. } t \in[0, T], \mathbf{x} \in \Omega .
\end{array}
$$

That is, for $j$-th neutron, optimal control $u^{j *}$

$$
\begin{array}{ll}
u^{j *}(t, \mathbf{x})=u^{j 1}, & \text { if } \boldsymbol{\nu}^{1}\left(\boldsymbol{u}^{*}\right)+\boldsymbol{\nu}^{2}\left(\boldsymbol{u}^{*}\right)>0, \text { a.e. } t \in[0, T], \mathbf{x} \in \Omega, \\
u^{j *}(t, \mathbf{x})=u^{j 2}, & \text { if } \boldsymbol{\nu}^{1}\left(\boldsymbol{u}^{*}\right)+\boldsymbol{\nu}^{2}\left(\boldsymbol{u}^{*}\right)<0, \text { a.e. } t \in[0, T], \mathbf{x} \in \Omega .
\end{array}
$$

This is so-called Bang-Bang principle of quantum optimal control $\boldsymbol{u}^{*}$ for many-body neutrons. 


\section{Conclusion and discussion}

Quantum optimal control for neutrons in nuclear reaction as target system had been surveyed in this work as a theoretic results. Physical control model has established for controlling of many-body neutrons collision in nuclear chain reaction of ${ }^{235} \mathrm{U}$. Theoretical full proof is completed for applying the quantum theory to mulitbody neutrons control system. It would be extended to control poly elementary particles in interaction or collisions.

Such a kind of theory, can be applied the a real-time nuclear reaction, or neutrons chain reaction? what the practical parameter should be taken to correspond the control input $u^{j}$ for $j$-th particle (neutron), the interacting coefficients $\mu=$ ? Initial guess $\psi_{0}^{j}=$ ? whether control theory to derive us to find a optimal solution $u^{j *}$ for neutron equation in quantum system (2). Theoretic answer is yes. Rest problems is left to our future works.

\section{Acknowledgments}

Thanks to ACS National Meeting \& Exposition for poster on collision.

\section{References}

[1] Adams R 1975 Sobolev Spaces (New York: Academic Press).

[2] Dautary R and Lions J L 1992 Mathematical Analysis and Numerical Methods for Science and Technology (Berlin-Heidelberg-New York: Springer-Verlag)

[3] Fursikov A V 2000 Optimal Control of Distributed Systems. Theory and Applications, Translations of Mathematical Monographs 187 (American Mathematical Society)

[4] Lions J L 1971 Optimal Control of Systems Governed by Partial Differential Equations (BerlinHeidelberg-New York: Springer-Verlag)

[5] Lions J L and Magenes E 1972 Non-Homogeneous Boundary Value Problems and Applications I II (Berlin-Heidelberg-New York: Springer-Verlag)

[6] Schrödinger E 1952 Nature 169538

[7] Temam R 1988 Infinite-Dimensional Dynamical Systems in Mechanics and Physis 68 (Applied Math. Sci.: Springer-Verlag)

[8] Wang Q F 2006 Proceeding of American Control Conference 1032

[9] Wang Q F 2008 European Science Foundation conference 'Chemical Control with Electrons and Photons' Poster

[10] Wang Q F 2009 IET Control Theory \& Applications 3(9) 1175

[11] Wang Q F and Nakagiri S (2009) Preprint of Annual Conference Japan Society for Industrial and Mathematics 59

[12] Wang Q F 2010 International Journal of Modern Physics E 19 (3) 393

[13] Wang Q F and Nakagiri S 2011 Applied Mathematics and Computations 2175695

[14] Wang Q F 2011 Practical Application of Optimal Control Theory: computational approach (Germany: Lambert Academic Publishing)

[15] Yukawa H 1935 Proc. Phys. Math. Soc. Jap. 1748 